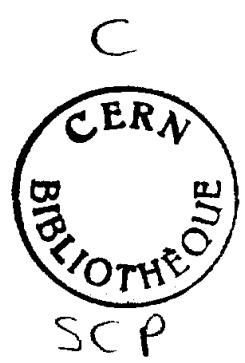

\title{
OPTO-ELECTRONIC DELAY FOR THE READOUT OF PARTICLE TRACKS FROM SCINTILLATING FIBRES( $\left.{ }^{*}\right)$
}

\author{
T. Gys (speaker), M. Garg ${ }^{(* *)}$, H. Leutz, F. Pengg and D. Piedigrossi \\ CERN, Geneva, Switzerland
}

\begin{abstract}
Due to the high event rate envisaged in future hadron colliders, the optoelectronic readout of a scintillating fibre tracker must pipeline optical images during the first-level trigger decision time. We describe in this paper the principle of a photon detector including a gatable opto-electronic delay line. Two prototypes of half scale have been tested, and preliminary results confirm the capability of these devices to delay optical images and select them by external trigger signals. Their measured time resolution amounts to $7 \mathrm{~ns}$ for a $400 \mathrm{~ns}$ delay. The space resolution, measured with cosmic rays detected by a hodoscope with a scintillating fibre target, is $110 \mu \mathrm{m}$ in a solenoid with only $0.03 \mathrm{~T}$ magnetic flux density. Finally, a readout scenario is discussed.
\end{abstract}

\section{INTRODUCTION}

Within the framework of the LAA Project [1], we are developing a compact scintillating-fibre tracking detector for future hadron colliders. It will be operated in a uniform magnetic field parallel to the beam axis. The tracker is made up of narrow-diameter scintillating fibres arranged in concentric, cylindrical shells surrounding the beam pipe (fig. 1). Table 1 summarizes, as an example, the detector lay-out described in the contribution of D'Ambrosio et al. [2].

The tracker covers, in this case, a total surface of $73.5 \mathrm{~m}^{2}$ while the total fibre cross-section to be readout is only $0.55 \mathrm{~m}^{2}$. The total fibre number is $1.610^{8}$. From the $\mathrm{X}$ section-to-surface ratio, we recognize the advantage of such a fibre arrangement in comparison to silicon strip (pixel) detectors, where the readout channel number is proportional to the covered surface.

(*) The work reported here is part of the LAA project.

(**) On leave from the Bergische Universität Wuppertal, Germany. 
Table 1

\begin{tabular}{|l|c|c|c|c|c|c|c|c|}
\hline & $\begin{array}{c}\text { Shell } \\
\text { radius } \\
(\mathrm{m})\end{array}$ & $\begin{array}{c}\text { Shell } \\
\text { thickness } \\
(\mathrm{cm})\end{array}$ & $\begin{array}{c}\text { H. shell(a) } \\
\text { length } \\
(\mathrm{m})\end{array}$ & $\begin{array}{c}\text { Shell(b) } \\
\text { X section } \\
\left(\mathrm{m}^{2}\right)\end{array}$ & $\begin{array}{c}\text { H. shell(c) } \\
\text { surface } \\
\left(\mathrm{m}^{2}\right)\end{array}$ & $\begin{array}{c}\text { X section-to-(d) } \\
\text { surface ratio } \\
\times 10^{-3}\end{array}$ & $\begin{array}{c}\text { Number(e) } \\
\text { of fibres } \\
\times 10^{6}\end{array}$ & $\begin{array}{c}\text { Number( } \\
\text { of tubes }\end{array}$ \\
\hline Shell \# 1 & 0.75 & 0.7 & 1.35 & 0.033 & 6.4 & 5.2 & 9.6 & 94 \\
Shell \# 2 & 1.00 & 1.4 & 1.80 & 0.088 & 11.3 & 7.8 & 25.6 & 63 \\
Shell \# 3 & 1.30 & 1.9 & 2.34 & 0.155 & 19.1 & 8.1 & 45.2 & 58 \\
Full tracker & & & & 0.552 & 73.5 & & 160.7 & 430 \\
\hline
\end{tabular}

Fibre diameter $=60 \mu \mathrm{m}$.

Fibre packing fraction $=90.7 \%$.

Packing fraction $=$ fibre core surface/fibre $($ core + cladding $)$ surface $=\frac{[\text { fibre diameter }]^{2}}{[\text { fibre diameter }+3 \mu \mathrm{m}]^{2}}$. (for hexagonal fibres separated by $3 \mu \mathrm{m}$ cladding).

(a) Half shell length $=$ shell radius $/ \tan 29^{\circ}\left(29^{\circ}=\right.$ zenith angle $\sim 1.35$ rapidity $)$.

(b) Shell $\mathrm{X}$ section $=2 \pi \times$ [shell radius $] \times$ [shell thickness $]$.

(c) Half shell surface $=2 \pi \times$ shell radius $\times$ half shell length $=2 \pi \times[\text { shell radius }]^{2} / \tan 29^{\circ}$.

(d) For each half shell.

(e) Number of fibres $=\frac{\text { shell } X \text { section }}{\text { fibre } X \text { section }}$.

Fibre $X$ section $=[\sqrt{ } 3 / 2] \times[\text { fibre diameter }+3 \mu \mathrm{m}]^{2}$.

(f) Number of tubes $=\frac{\text { shell } X \text { section }}{\text { tube active area }}$.

The tube active areas are $3.5,14.0$ and $26.6 \mathrm{~cm}^{2}$ for shells \# 1,2 and 3 respectively.

\section{ROLE OF THE DELAY TUBE IN THE TRIGGER SEQUENCE}

In future hadron colliders, the expected time needed to process and transmit the first-level trigger signals to the gates of the opto-electronic chains will be $\geq 1 \mu \mathrm{s}$ and therefore much longer than the 15 ns between two successive bunch crossings. Consequently, optical images produced by our tracker must be pipelined. For that purpose, we designed an opto-electronic device able to delay in a controlled way those images, and to select and amplify a small fraction of them [3]. Assuming a rejection capability of $10^{3}$ for the first-level trigger, the readout frequency of the opto-electronic delay tube is reduced from $67 \mathrm{MHz}$ to $67 \mathrm{kHz}$. 


\section{BASIC SCHEME OF THE DELAY TUBE}

The proposed delay tube is a $600 \mathrm{~mm}$ long, electromagnetically focused image intensifier. The tube is divided in 6 sections separated by 5 grids labelled G1 to G5 (fig. 2). The requirements stated in the previous paragraph are fulfilled in the following way:

- track points coming from the scintillating fibres are detected by a photocathode and converted into photoelectrons;

- the delay is achieved by to and fro drifts of very low-energy photo-electrons in the first four sections of the tube;

- space resolution of the track points is ensured by the detector magnetic field, parallel to the photoelectron trajectories;

- the required time resolution is obtained by compensating the inevitable velocity spread of the photoelectrons with different drift lengths (see next paragraph);

- the gating is realized by blocking the photocathode before applying a 5 ns negative pulse on grid $\mathrm{G} 1$;

- selected track points are accelerated (at $30 \mathrm{kV}$ ) before hitting a silicon pixel array;

- fast track reconstruction is provided by random access readout of the hit pixels.

\section{THE BUNCHING PRINCIPLE}

Electrons are emitted from a photocathode with complex angular and energetic distributions. In case of blue incident light and with bialkali photocathodes, the energy of an emitted photo-electron can range from 0 to $1.8 \mathrm{eV}$, according to the work function of the photocathode. Without additional bunching of the electronic images, fast photo-electrons of the actual image will catch up slow ones of the previous images.

In the delay tube, the bunching is ensured by the reflex klystron principle (sect. 17 of ref. [4]). After their emission, electrons are accelerated by G1 and drift at constant velocity $(\approx 1 \mathrm{~m} / \mu \mathrm{s})$ between $\mathrm{G} 1$ and $\mathrm{G} 3$ (G1, G2 and G3 being at the same potential of a few volts above the cathode potential). A reflector grid G4, operated below cathode potential, makes the electrons return to G1. Since the faster electrons penetrate farther into the reflection region and therefore take a longer path to return, all the electrons belonging to the same image finally form in bunches. The condition for two electrons of emission energy $\mathrm{e} \times \mathrm{V}_{01}$ and $\mathrm{e} \times \mathrm{V}_{02}$ (with $\mathrm{V}_{01}<\mathrm{V}_{02}$ ) to bunch within the selection section can be found with a mechanical analogy :

$$
\frac{D}{d}=2 \times\left(\frac{V_{01}+V_{G}}{V_{02}+V_{G}}\right)^{1 / 2} ;
$$

where $\mathrm{D}$ is the length of the selection and zero electric field sections, $d$ is the distance travelled by the fastest electron in the reflection section, and $V_{G}$ (in volts) is the voltage applied at grids G1, G2 and $\mathrm{G} 3 . \mathrm{V}_{01}$ and $\mathrm{V}_{02}$ are expressed in volts. In this approach, the influence of the transit time in the acceleration section has been neglected. Starting from the above condition, we designed, assisted by computer simulations, a tube with a time resolution of less than $10 \mathrm{~ns}$.

\section{THE IMAGE ELIMINATION MODE}

The delay tube works in "image elimination mode" as long as the first-level trigger is not active: in this case, every image arriving on the cathode must be finally discarded. After their to and fro drift, the electrons are slowed down between G1 and the cathode, and are finally absorbed by the cathode. 


\section{THE IMAGE SELECTION MODE}

The selection process of one image, actuated by the first-level trigger, can be decomposed in three phases:

\section{Phase 1}

Just before the reflected image to be selected enters from G2 the selection zone, the cathode voltage is increased (e.g. from $0 \mathrm{~V}$ to $10 \mathrm{~V}$ ) in order to prohibit any subsequent photo-electron from being emitted during the selection process. The selection zone is thus gradually cleared from forwarddrifting electrons.

\section{Phase 2}

As soon as the triggered image is completely confined between G1 and G2, a 5 ns negative $(-200 \mathrm{~V})$ pulse is applied on grid G1. Most of the electrons inside the selection zone acquire a large velocity $(10 \mathrm{eV})$ and are sent back to $\mathrm{G} 3$. At the same time, the photocathode is grounded, to provide again new track pictures.

\section{Phase 3}

The delay tube runs again in image elimination mode while the selected electrons due to their $10 \mathrm{eV}$ energy cross the reflection and the screening zones, and are finally accelerated by the high voltage $(30 \mathrm{kV})$ applied on the readout end, where they are detected. The duration of the positive voltage applied on the photocathode determines the dead time of the tube. It is of the order of $40 \mathrm{~ns}$ (i.e. $\leq 3$ bunch crossings).

\section{TIME RESOLUTION MEASUREMENT}

Two $300 \mathrm{~mm}$ long prototypes (scale 2:1) have been manufactured by DEP B.V. (Roden, The Netherlands) (fig. 3). They include all the elements schematically depicted in fig. 2 . The readout is not yet a pixel array as finally foreseen, but a fast phosphor screen (P47). The way we define the time resolution $\delta t$ of the delay tube refers to its ability to keep optical images, initially adjacent in time, with as less overlap as possible after the delay, i.e. at the time of the selection. Since $\delta$ t adds up to the cell busy time of the scintillating fibre detector and may affect its occupancy (see relation (12) of ref. [2]), the estimation of this parameter is of crucial importance. We have measured it in two different ways.

The first experimental procedure is represented in figure 4.a. The light source is a blue $\mathrm{L}^{\text {? }}$ pulse (peak wavelength $=420 \mathrm{~nm}, \mathrm{FWHM}=4 \mathrm{~ns}$, FW at $10 \%$ of the maximum amplitude $=$ $12 \mathrm{~ns}$, FW at $5 \%$ of the maximum amplitude $=19 \mathrm{~ns}$ ). In our experiment, the origin of time is defined by the start of the LED pulse. The delay tube is working in selection mode and supplied with the following voltages : the cathode at $-6.8 \mathrm{~V}$, grids $\mathrm{G} 1, \mathrm{G} 2, \mathrm{G} 3, \mathrm{G} 5$ at $0 \mathrm{~V}$, grid $\mathrm{G} 4$ at $-8.1 \mathrm{~V}$ and the phosphor screen at $+6 \mathrm{kV}$. In these conditions, we achieve a time delay of the light pulse of $400 \mathrm{~ns}$. The selection pulse is triangular, $-180 \mathrm{~V}$ in amplitude, with fall and rise times of $3 \mathrm{~ns}$. The time when the selection pulse is triggered is scanned from $380 \mathrm{~ns}$ to $410 \mathrm{~ns}$ in $10 \mathrm{~ns}$ steps. The selected signal, visible on the phosphor screen, is detected via a light guide by a fast photomultiplier (Hamamatsu R1635). Figure 5 shows the intensity peak of the selected signal versus the position in time of the selection pulse. This peak (FWHM $=17 \mathrm{~ns}$ ) is the result of the convolution of three contributions : the first is the input light pulse (FWHM =4 ns). The second is the integration window of the selection process : all photoelectrons confined between G1 and G2 are selected (*).

$\left(^{*}\right)$ The selection zone has been designed in such a way that all optical signals coming from the scintillating fibre tracker and belonging to one bunch crossing are selected. Taking into account the decay time of the scintillator and the travel time in the fibres, the total signal duration can be estimated. The selection section length must be bigger than the product of this duration by the average photoelectron velocity in between $\mathrm{G} 1$ and $\mathrm{G} 2$. 
The corresponding integration time is thus given in a good approximation by the selection section length $(0.025 \mathrm{~m})$ divided by the average photoelectron velocity in this section $(7.8 \mathrm{eV})$, i.e. $15 \mathrm{~ns}$. The third contribution is the temporal spread due to the non-perfect bunching process, representing the intrinsic time resolution $\delta t$ of the delay tube. We can therefore write :

$$
(17 n s)^{2}=(4 n s)^{2}+(15 n s)^{2}+(\delta t)^{2}
$$

The above relation results in $7 \mathrm{~ns}$ for the intrinsic time resolution $\delta \mathrm{t}$.

The second experimental procedure is more qualitative (fig. 4.b). It uses another light source made up of 9 red LEDs. Each LED illuminates a different area of the photocathode of the delay tube, and is pulsed every $135 \mathrm{~ns}$ with a delay of $15 \mathrm{~ns}$ one with respect to another. This means that LED \# 0 is pulsed at time $t=0$, LED \# 1 at $t=15 \mathrm{~ns}$, and so on until $t=135 \mathrm{~ns}$ where the sequence starts again. The LED pulses are very short : FWHM $=4 \mathrm{~ns}, \mathrm{FW}$ at $10 \%$ of the maximum amplitude $=6 \mathrm{~ns}$, FW at $5 \%$ of the maximum amplitude $=10 \mathrm{~ns}$. The tube is supplied in the same way as before. The selection process is not changed either. The light signal on the phosphor screen is amplified in order to be detected by a standard CCD device. For this amplification, we use a gated microchannel plate (MCP) image intensifier. The resulting image is acquired by a computer and analyzed. With this set-up, we are able to select a determinate LED out of the $67 \mathrm{MHz}$ sequence of LED pulses. This is clearly visible on figure 6 where LED \# 7 is selected. Two background noises can be seen : the uniform noise comes from the selection pulse interferences picked up by the CCD driver. This noise is only dependant on the experimental procedure and will be suppressed in future tests. The non uniform noise represents the tiny fraction of light coming from the other LEDs, mainly due to their long decay time constant selected together with LED \# 7 (this is the reason why this noise decreases from LED \# 6, 5, etc. down to LED \# 8).

\section{SPACE RESOLUTION MEASUREMENT}

We have measured the space resolution of the optoelectronic chain by detecting cosmic rays with a scintillating fibre target. The experimental set-up is represented in fig. $7:$ the fibre target is $1 \mathrm{~m}$ long, 10 (horizontal) $\times 5$ (vertical) $\mathrm{mm}^{2}$ in cross-section, and is composed of $60 \mu \mathrm{m}$ hexagonal fibres (fig. 8). Two bulk scintillator bars, $50 \mathrm{~cm}$ long and $1 \times 1 \mathrm{~cm}^{2}$ in cross-section, are placed above and below the target and used as trigger detectors. The resulting angular acceptance of this hodoscope is typically $\pm 5^{\circ}$. The ends of the scintillator bars are connected with fast photomultipliers whose signals are, after discriminator shaping, put in coincidence. The fibre target is placed in contact with the photocathode of the delay tube. The optoelectronic chain, composed of the delay tube, the MCP tube and the CCD is identical to the one used for the time resolution measurement. The magnetic flux density, provided by a small solenoid, is only $0.03 \mathrm{~T}$.

Whenever trigger coincidences are received (typically every 15 seconds), the CCD image of the cosmic ray track is acquired. After CCD background subtraction and data compression, the image is stored on computer disk. One data taking run consists of 500 cosmic events. For every event, a cluster finding algorithm determines the position and intensities of the hits (a hit is a photon from the scintillating fibres detected by the photocathode of the delay tube). These data are then used to make the least square fit of the track with a straight line. The residual positions of the clusters with respect to the fitted track are computed. Examples of cosmic events along with their straight line fit are displayed in figures 9.a to 9.d. The histogram of the residuals for 5000 tracks is represented in fig. 10. The dashed curve, superimposed on the histogram, represents a gaussian distribution with the same maximum and FWHM as the histogram. In this plot, the few events with only 1 or 2 hits and all tracks outside the angular acceptance of the hodoscope are excluded. There is no particular cut on the kind of events, which may thus include not only the wanted hard but also the soft component of the cosmic ray flux, as well as environmental background. These contributions are, together with backscattering at the MCP phosphor screen (fig. 7) and the CCD noise, responsible for the tails deviating from the gaussian. 
The two-track resolution, defined as the full width at half maximum of the distribution of the residuals, is $110 \mu \mathrm{m}$. The space resolution can be separated in two parts : the intrinsic resolution of the scintillating fibres $(60 \mu \mathrm{m}$ [5]) and the space resolution of the optoelectronic chain $(\approx 90 \mu \mathrm{m})$, which results from the following:

- the magnetic flux density of $0.03 \mathrm{~T}$ is definitely too low. As an example, for an electron emitted from the photocathode with a transverse velocity of $1 \mathrm{eV}$, the curling diameter is $226 \mu \mathrm{m}$ in $0.03 \mathrm{~T}$;

- in the solenoid we use for our tests, the uniformity of the magnetic field is very bad (the ratio $\Delta \mathrm{Bz} / \mathrm{Bz}$ reaches up to $10 \%$ ) and image distortions exist;

- transverse electric fields at the level of grid G5 of the delay tube induce ExB effects;

- the MCP image intensifier and the CCD following the delay tube degrade the global resolution of the chain.

The curling radius and the ExB drift are effects scaling with $1 / \mathrm{B}$ and can be drastically reduced if we use a stronger magnetic field. Moreover, the replacement of the phosphor screen of the delay tube by a silicon pixel device directly bombarded with photoelectrons will make the subsequent MCP tube and $\mathrm{CCD}$ obsolete. The space resolution will improve accordingly.

\section{READOUT SCENARIO}

The choice of the delay tube solution for an analog pipeline has the following advantages:

- It is an imaging device by itself, and can perform parallel processing of up to $410^{5}$ microfibres. As a consequence, it yields a low cost per fibre channel.

- It preserves both excellent time resolution (within the bunch crossing period) and space resolution (within the microfibre diameter).

- It has a low power consumption (of less than $1 \mathrm{~W}$ per tube) and thus minimizes heat production.

- It represents only 2.4 percent of a radiation length.

- It is expected to be radiation hard.

Assuming a rejection capability of $10^{3}$ for the first-level trigger, the mean image rate at the delay tube output is in the range of $100 \mathrm{kHz}$. The readout is a silicon pixel device directly bombarded with the photo-electrons of the selected images. The mean energy to create an electron-hole pair in this mode is $3.6 \mathrm{eV}$. With a photo-electron energy of $30 \mathrm{keV}$, we expect a pixel signal . $\sim 8.310^{3}$ electrons. The fibre tracker occupancy has been estimated to be of the order of $0.1 \%$ [2] for a luminosity of $10^{34} \mathrm{~cm}^{-2} \mathrm{~s}^{-1}$, which leads to read out about 10 hits per square centimeter of silicon. In this scenario, since pixel devices are randomly accessed, their readout time will take only a few microseconds.

The application of this technology becomes accessible due to the development of silicon pixel devices for direct particle detection. In this context, it is worthwhile noting that the total pixel surface required by our fibre tracker is much smaller than the one needed for direct particle detection as indicated in table 1. 


\section{CONCLUSIONS}

For the readout of a scintillating fibre tracker, a novel opto-electronic delay line has been designed, providing image delay, selection and amplification. It consists of an electro-magnetically focused image intensifier working with low-energy photo-electrons. Experimental results show encouraging performances in terms of optical signal delay, selection and amplification capabilities.

We investigated in detail the time resolution of the device by testing the selection of a single blue LED light pulse and a red LED light pulse out of several consecutive pulses separated by $15 \mathrm{~ns}$. We reach a time resolution of $7 \mathrm{~ns}$ for a $400 \mathrm{~ns}$ delay. The space resolution has been measured with cosmic rays, and is $110 \mu \mathrm{m}$ for a $300 \mathrm{~mm}$ long tube in a $0.03 \mathrm{~T}$ magnetic flux density, which will inevitably improve at higher magnetic fields as foreseen in future LHC-detectors. In parallel, we are looking for the replacement of the delay tube phosphor screen by an electron-bombarded silicon detector.

\section{Acknowledgement}

We want to thank Prof. A. Zichichi for his continuous interest and support for our work. We also acknowledge substantial help from P. Nappey and J. Dupont.

\section{REFERENCES}

[1] A. Zichichi, The LAA Project, ICFA Instrum. Bull. 3 (1987) 17.

[2] C. D'Ambrosio et al., Optimization of central paricle tracking at future hadron colliders, CERN-PPE/92-69, 30 March 92.

[3] T. Gys et al., Optoelectronic delay for the readout of particle tracks from scintillating fibres, in New Technologies for Supercolliders, edited by L. Cifarelli and T. Ypsilantis, Plenum Press Publishing Corp. (1991), vol. 57, Physical Sciences.

[4] K. Spangengerg, Vacuum tubes, Mc Graw-Hill (1948).

[5] C. D'Ambrosio et al., Imaging performance obtained with fused bundles of scintillating fibres, CERN-PPE/92-108, 2 July 92. 


\section{FIGURE CAPTIONS}

Fig. 1 Arrangement of scintillating fibre shells for tracking within the free barrel region of the calorimeter. In this case, the tracker covers up to 1.35 rapidity. Readout with electromagnetically focused delay tubes is at $z=0$ of each shell. $\theta$ means the polar angle.

Fig. 2 Basic scheme of the delay tube. The indicated voltages are typical values.

Fig. 3 Photograph of the DEP prototype. This tube is a half scale model.

Fig. 4 Experimental set-ups used for the time resolution measurements of the delay tube working in image selection mode :

a. The light source is a blue LED. The selected signal on the phosphor screen is detected by a fast photomultiplier.

b. The light source is composed of nine red LED pulses separated by $15 \mathrm{~ns}$. The selected signal on the phosphor screen is amplified by an MCP image intensifier and detected by a CCD.

Fig. 5 Intensity of the signal resulting from the selection of a blue LED pulse (fig. 4.a) delayed b3 $400 \mathrm{~ns}$. The horizontal axis is the position in time of the selection pulse.

Fig. 6 Selection of a red LED pulse (fig. 4.b) out of a sequence of pulses separated by $15 \mathrm{~ns}$. Visible background from the non selected LEDs mainly arises from the long term component of the LED pulses.

Fig. 7 Experimental set-up used for the space resolution measurement with cosmic rays.

Fig. 8 Magnified view of the cross-section of the scintillating fibre target used for the detection of cosmic rays. The fibre "diameter" is $60 \mu \mathrm{m}$. Each fibre bundle is $2.5 \times 2.5 \mathrm{~mm}^{2}$ in cross-section, including a surrounding PMMA cladding $50 \mu \mathrm{m}$ thick. A cushion distortion of the bundles is also visible (it is no longer present with the newer bundles). The sensitive area of the target cross-section is estimated to be less than $90 \%$ of the total cross-section.

Fig. 9 Figures 9.a to 9.d are examples of cosmic events. Their least square fit with a straight line is also displayed along with a corridor of $\pm 55 \mu \mathrm{m}$.

Fig. 10 Histogram of the residual position of hit clusters with respect to the fitted straight line of cosmic ray tracks. The dashed line represents a gaussian distribution with the same maximum and FWHM as the histogram. The background tails arise from soft component and environmental contributions, backscattering at the MCP phosphor screen (fig. 7) and CCD noise. 


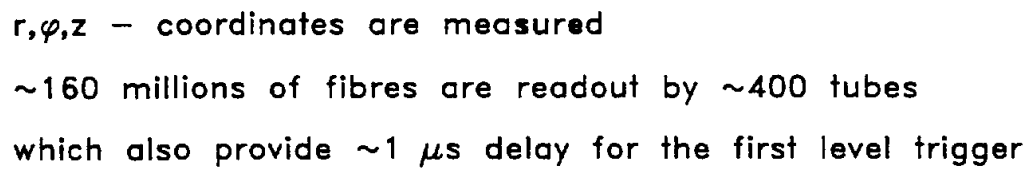

Polar- $(r, z)-$ plane

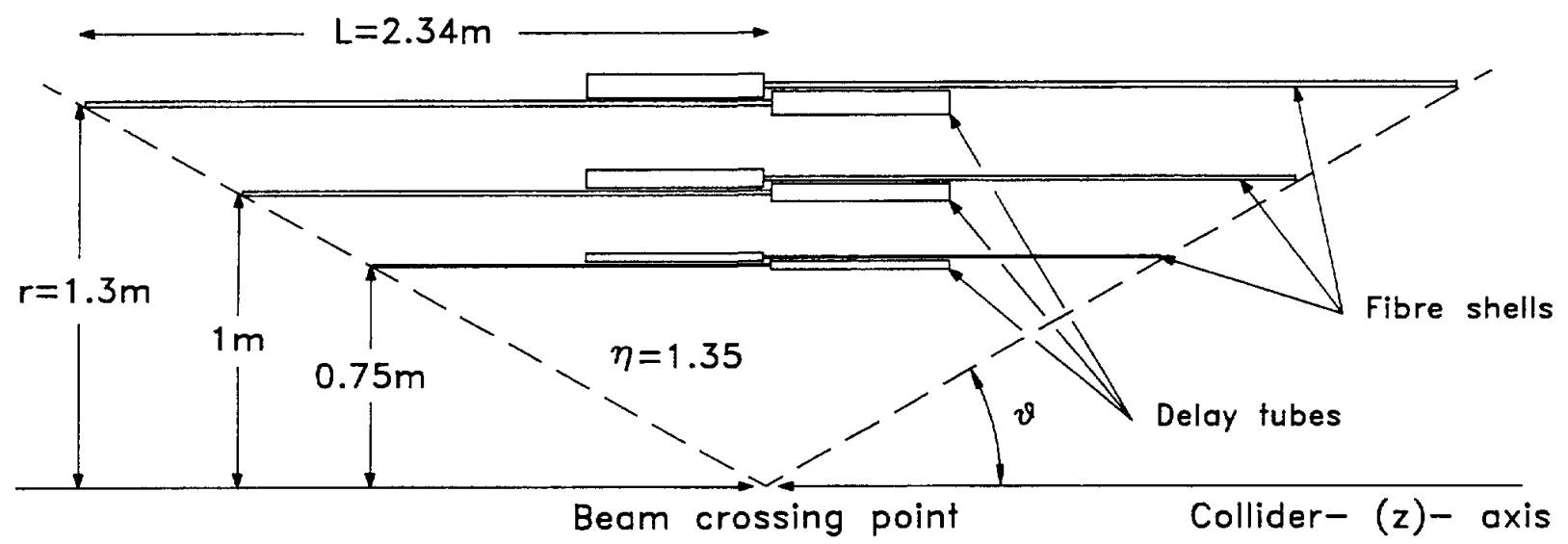

Fig. 1

LENGTH: $60 \mathrm{~cm}$

PHOTOCATHODE
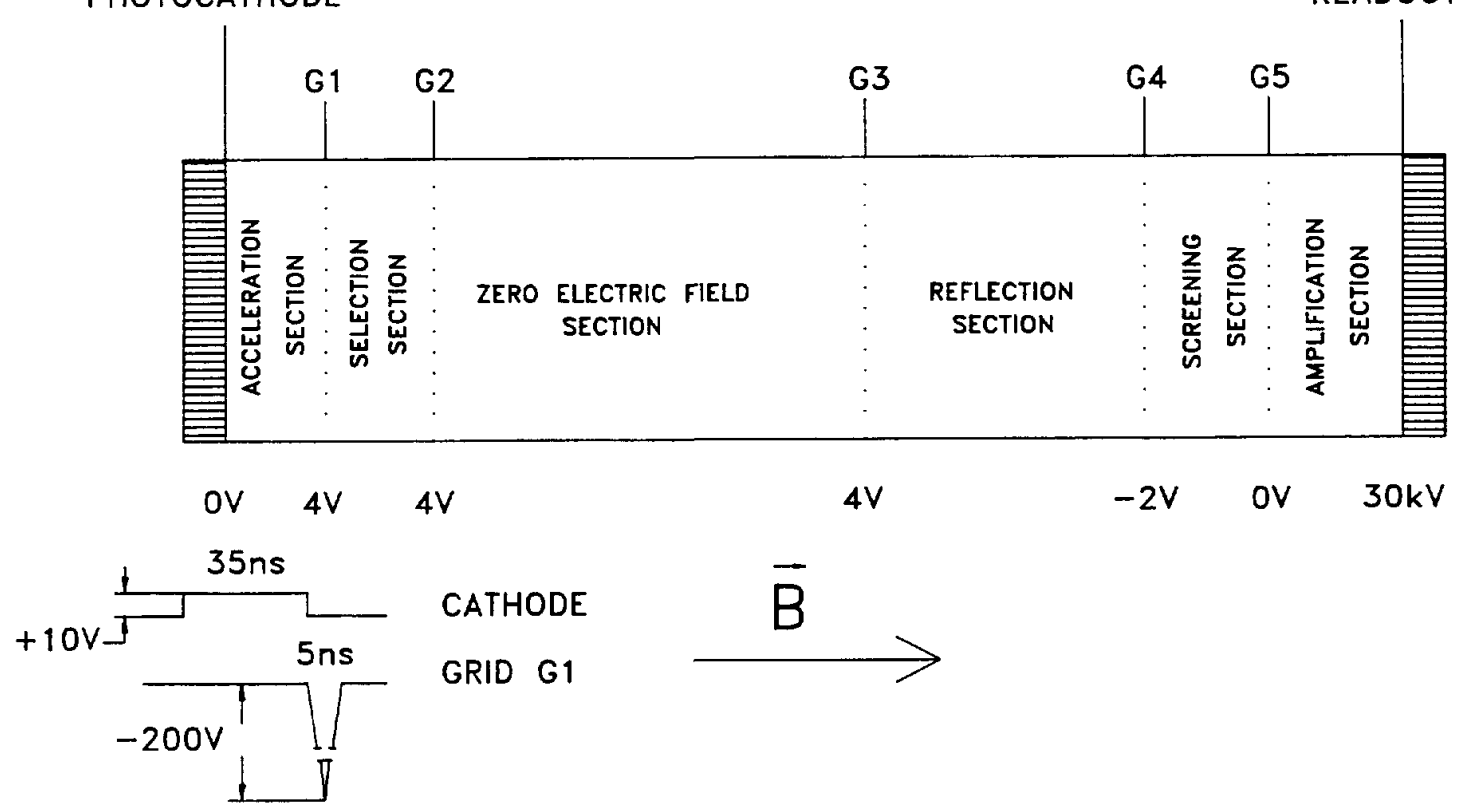

Fig. 2 


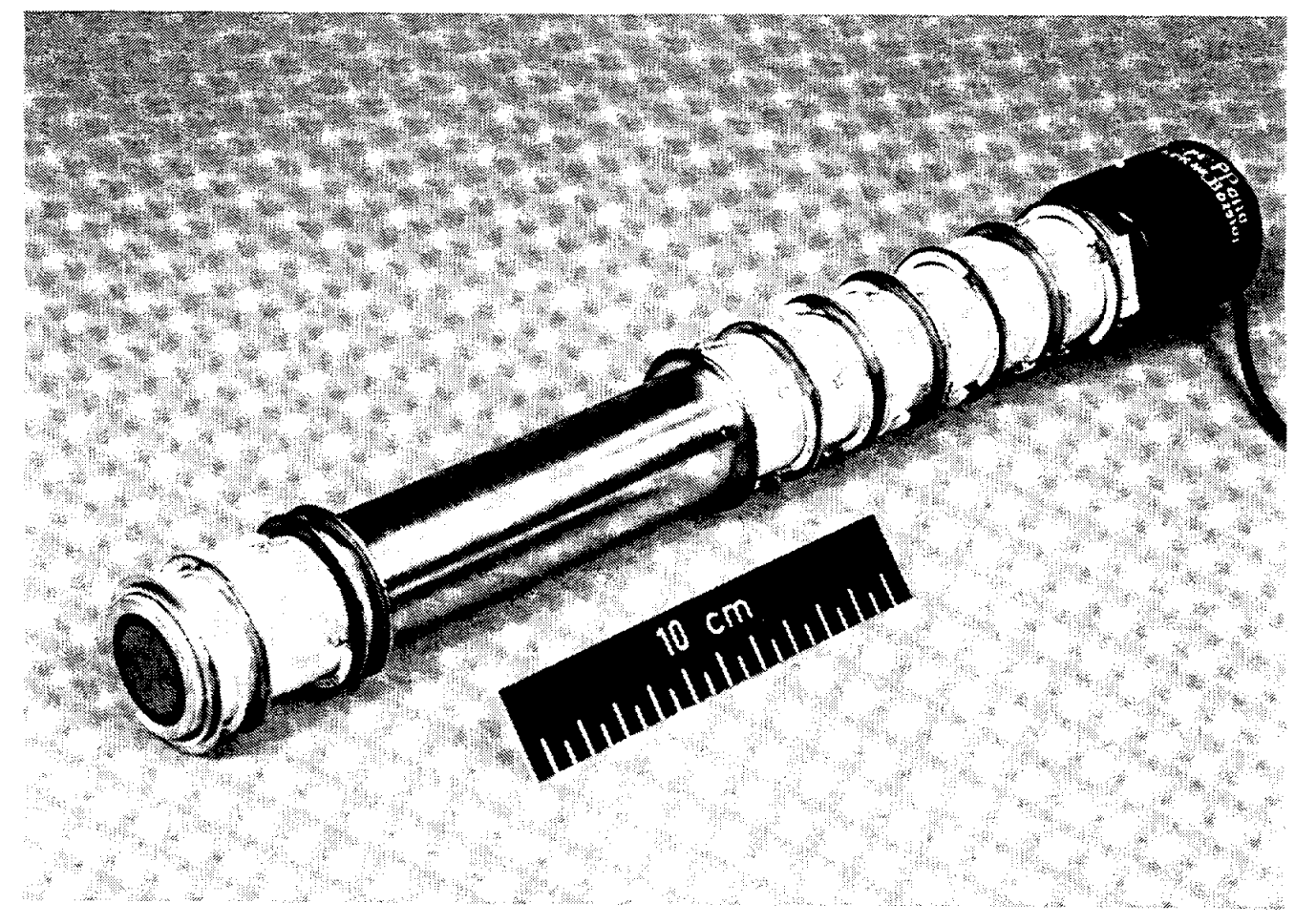

Fig. 3 


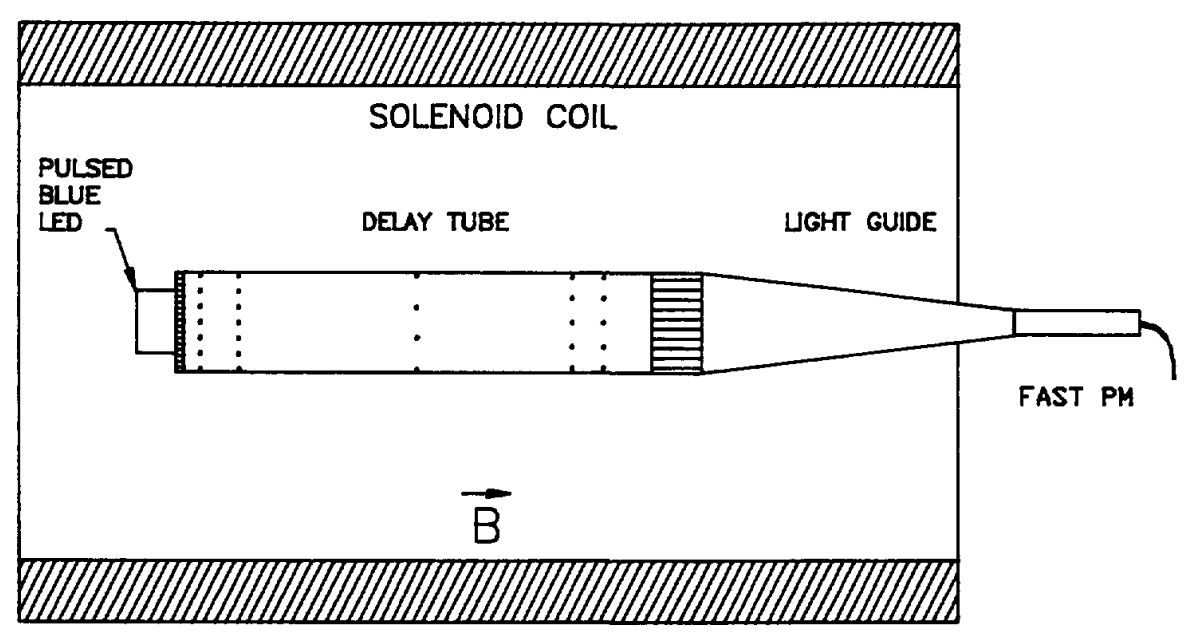

Fig. 4.a

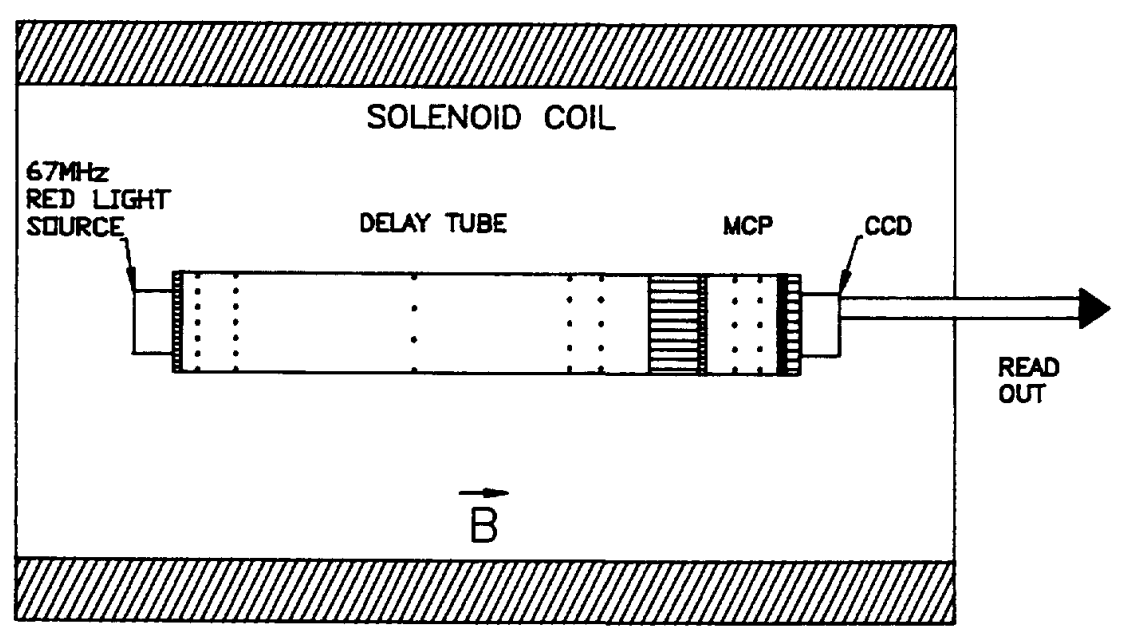

Fig. 4.b 


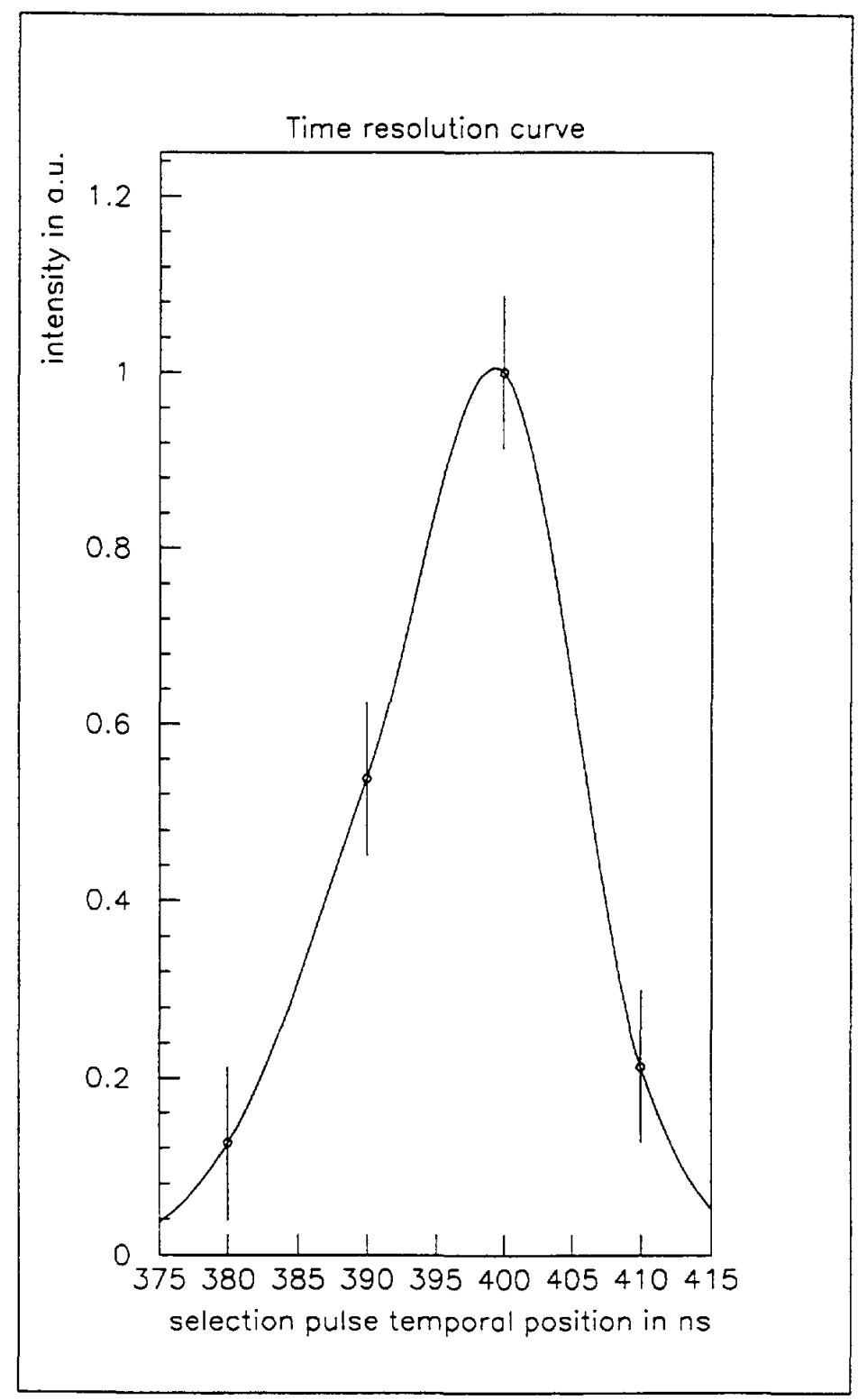

Fig. 5 


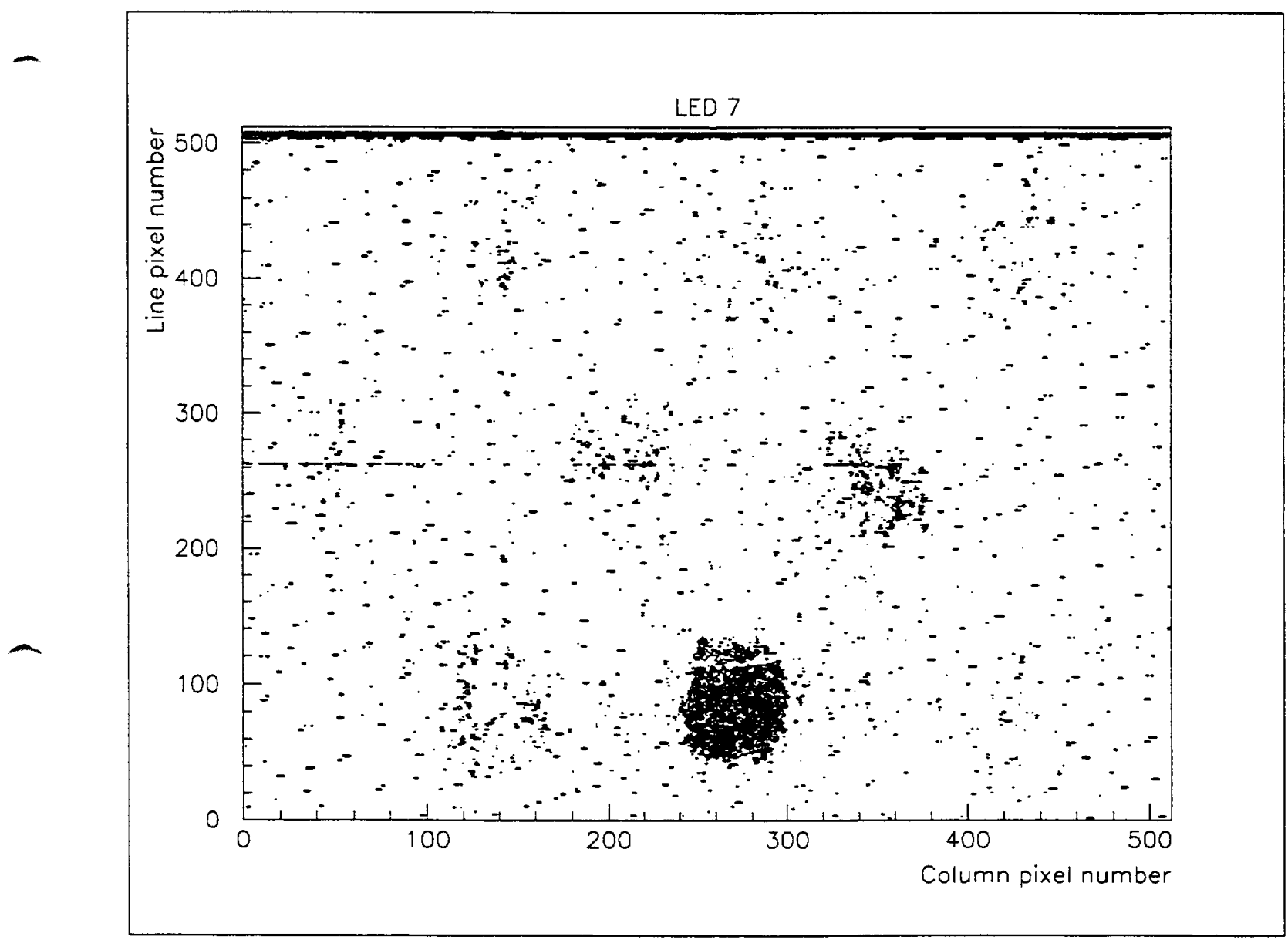

Fig. 6 


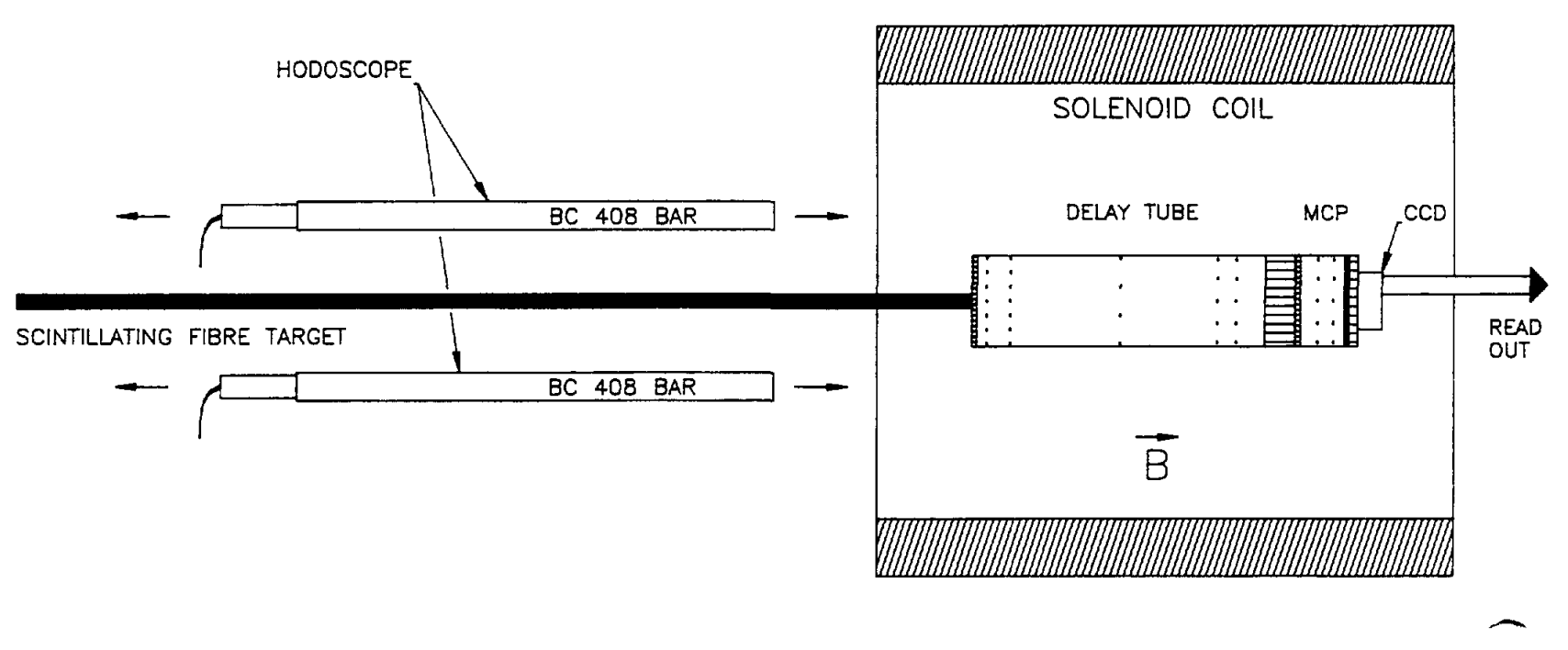

Fig. 7 


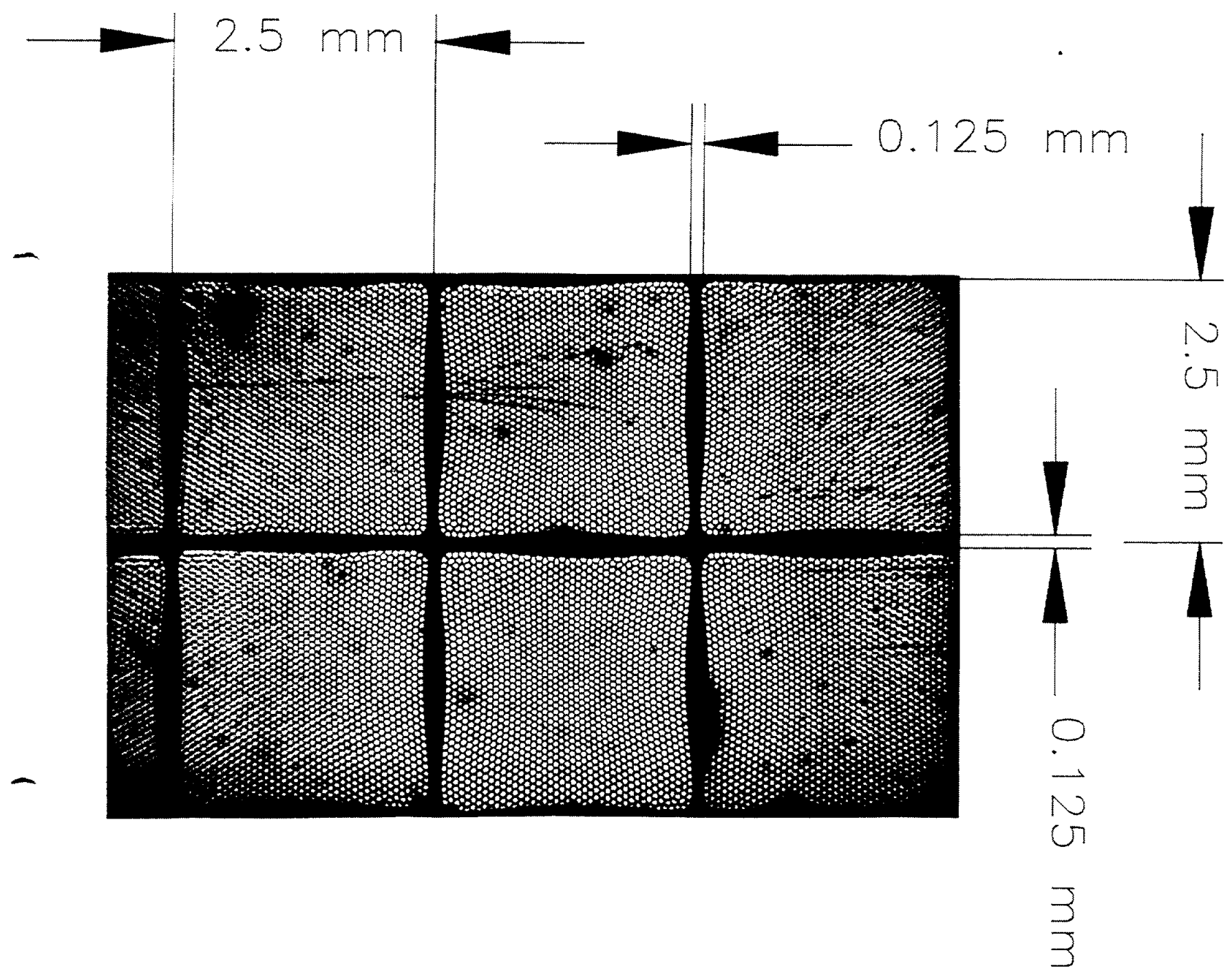

Fig. 8 


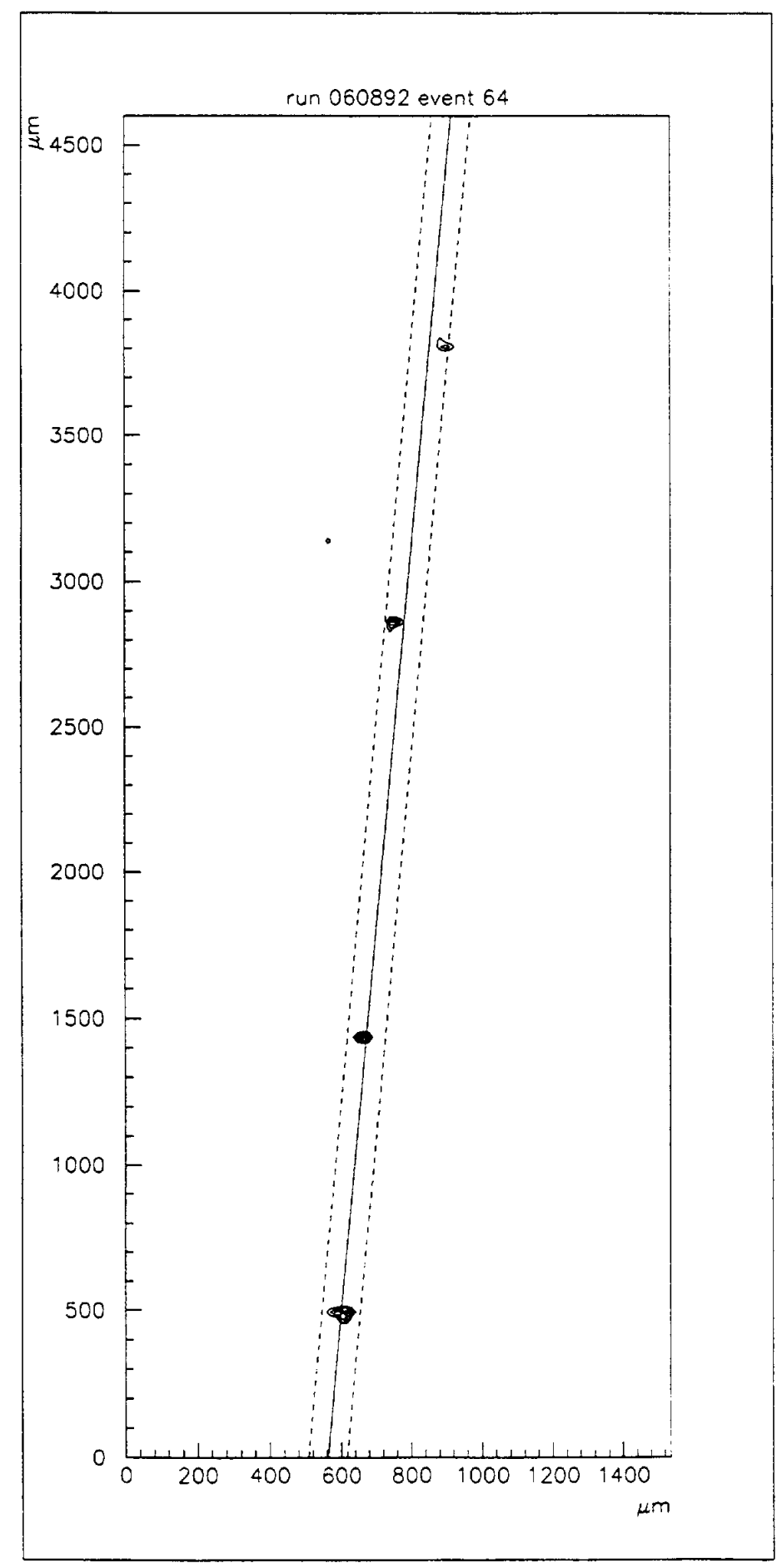

Fig. 9.a 


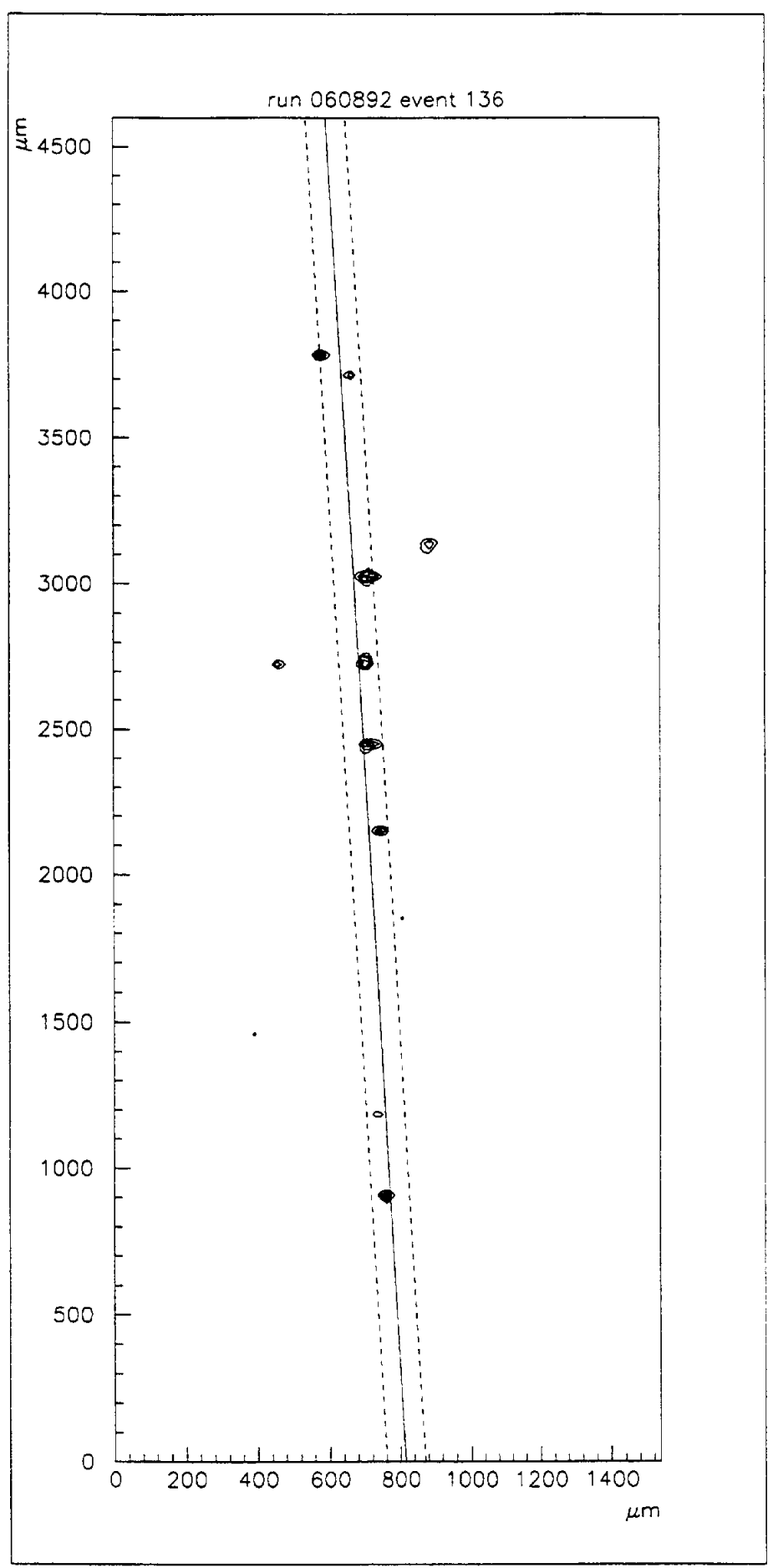

Fig. 9.b 


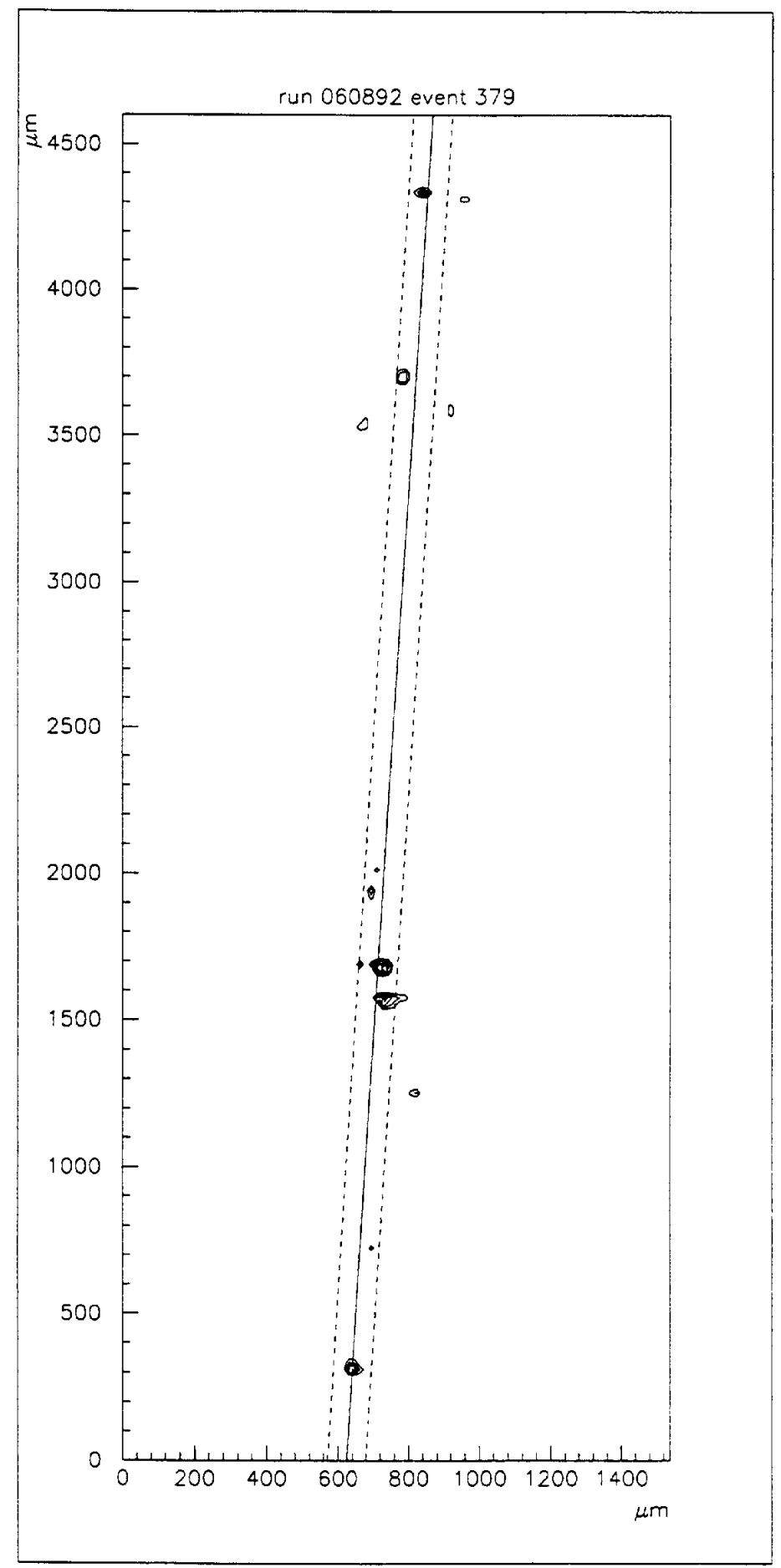

Fig. 9.c 


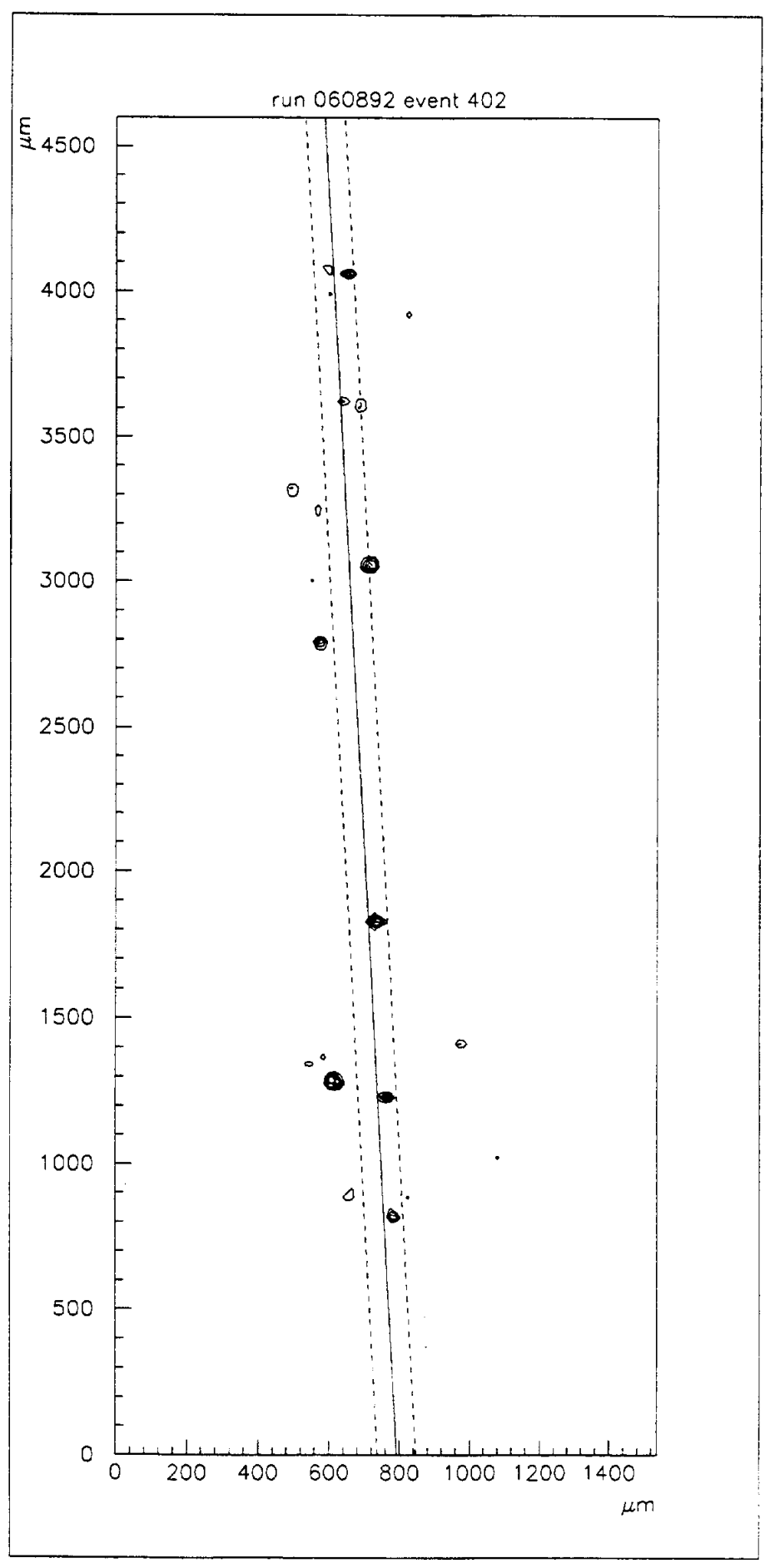

Fig. 9.d 


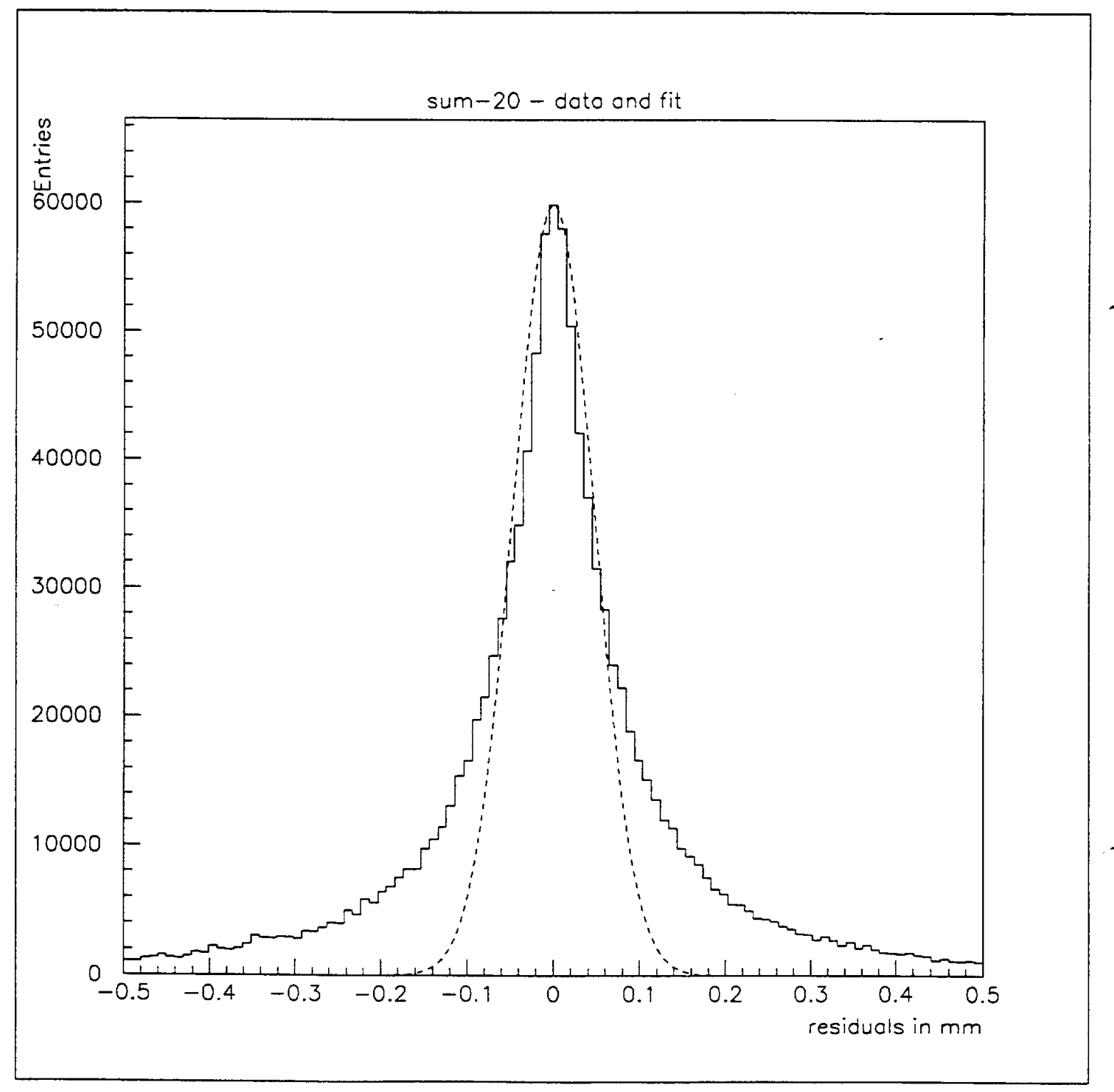

Fig. 10 